\title{
Femtosecond Laser Structuring of Materials: A Review
}

\author{
George Amoako ${ }^{1}$ \\ ${ }^{1}$ Department of Physics, University of Cape Coast, Cape Coast, Ghana \\ Correspondence: George Amoako, Department of Physics, University of Cape Coast, Cape Coast, Ghana. E-mail: \\ gamoako@ucc.edu.gh
}

Received: February 22, 2019

Accepted: March 24, 2019

Online Published: April 3, 2019

doi:10.5539/apr.v11n3p1

URL: http://dx.doi.org/10.5539/apr.v11n3p1

\begin{abstract}
In this review the interaction between femtosecond laser and matter with emphasis on silicon and some metals have been appraised. For silicon, spikes are produced as a result of interaction in the presence of background gas such as $\mathrm{SF}_{6}$, or $\mathrm{H}_{2} \mathrm{~S}$ with an appropriate background pressure, but spikes are not formed in the presence $\mathrm{N}_{2}$ or air. The formation of the spikes increase the infrared light absorption of silicon to near unity. Interaction of femtosecond laser with metals form periodic surface structures that make the metals exhibit many different morphological characteristics. This processing technique allows the creation of a variety of colors on a metal that ultimately lead to control of its optical properties from UV to terahertz. From the assessment made, the potential of femtosecond laser structuring of materials for cost-effective nano-manufacturing is high.
\end{abstract}

Keywords: Femtosecond Laser, Interaction, Plasma, Microstructure, Metals

\section{Introduction}

For almost 50 years scientists have studied the interaction of laser with matter, and over the past several decades progress in precision control of pulse repetition rate and carrier-envelope phase of ultrafast lasers has established a strong and interesting research into materials removal. The interaction of a high power laser beam with material has many applications including chemical analysis, micromachining and pulse laser deposition of thin films. The laser-material interaction involves complex processes of heating, melting, vaporization, ejection of atoms, ions and molecules, shock waves, plasma initiation and plasma expansion. Generally, the electron-lattice relaxation time in metals is 1-10ps (Brorson et al., 1990; Eesley, 1986) and this makes the mechanism of femtosecond laser ablation different from that of longer pulsed laser ablation in metals. This allows the local structuring of bulk transparent materials without damage to the bulk. Thermal ablation occurs when the laser pulse width is longer than the electron-lattice relaxation time. A variety of micrometer scale surface patterns have been observed on silicon surfaces after pulsed laser irradiation (Dolgaev et al., 2001; Fauchet \& Siegman, 1982; Her et al., 1998; Her et al., 2000; Pedraza et al., 1999; Rothenberg \& Kelly, 1984). Femtosecond laser ablation is the process of low-threshold-material-removal from surfaces governed by plasma formed at the surface of the material via generation of extreme temperatures and pressures excited via multi-photon absorption. The transfer of plasma energy through the material is caused through lattice expansion and bond breaking. Since the time scale of the laser is sub-picosecond and all the accompanying processes are on this scale, thermal diffusion into the material is nearly nonexistent and material does not have enough time to melt and solidify. Absorbed laser energy is fully deposited into the target and higher efficiency of material ablation can be obtained (Le Drogoff et al., 2001). As an example, the ablation physics in femtosecond laser applications is drastically different from those in conventional nanosecond pulsed laser ablation (Crouch et al., 2004). Ultrashort laser irradiation technologically offers an alternative method for annealing ion-implanted semiconductors (Kanemitsu et al., 1985; Nissim et al., 1983).

Several authors have reported in the last four decades on the formation of very smooth holes, cavities and trenches by femtosecond laser pulse irradiation (Kautek \& Krueger, 1995; Momma et al., 1997) and structuring of the surfaces of semiconductors, metals and dielectrics (Allmen \& Lau, 1982; Dam et al., 1996; Fauchet \& Siegman, 1982; Jhee et al., 1985; Young et al., 1983). Mazur's group have reported formation of sharp spikes on a 'black' silicon surface without using masks (Her et al., 1998; Kautek \& Krueger, 1995) and using masks (Shen et al., 2003) due to irradiation with femtosecond laser pulses in the presence of $\mathrm{SF}_{6}$ or $\mathrm{Cl}_{2}$. They (Tull et al., 2006) have also shown that in the presence of a background gas, either hydrogen $\left(\mathrm{H}_{2}\right)$ or hydrogen sulfide $\left(\mathrm{H}_{2} \mathrm{~S}\right)$, two phases of silicon exist in the plume material and also irradiation of silicon in water results in the formation of nanoscale rods 
(Shen et al., 2008). Other authors have also reported the structuring of metal films on substrates (Ko et al., 2006) and bulk metal surfaces (Hirayama \& Obara, 2002; Nedialkov et al., 2007; Vorobyev \& Guo, 2006, 2008; Wang \& Guo, 2006; Wellershoff et al., 1999) and creating 'black metals' (Vorobyev \& Guo, 2008). The structuring of the surfaces of transparent materials by femtosecond laser that can locally induce structural and chemical changes in the bulk of the material has several applications including increased light absorption (Crouch et al., 2004; Sheehy et al., 2007; Vorobyev \& Guo, 2005; Vorobyev et al., 2009), bulk heating (Schaffer et al., 2003), chemical catalysis (Scott et al., 2003), and colorizing metals (Vorobyev \& Guo, 2008).

This review discusses the latest developments in femtosecond laser structuring with emphasis on sharp spike formation on silicon and structuring on metals. It also looks at the increased light absorption after structuring of these materials with focus on work done by the Mazur and Guo groups. It will be restricted to highlights over the last 30 years. The paper will be organized in three sections as follows: Section (1) will be introduction, Section (2) will comprise the structure formation and optical properties with Section (3) being summary.

\section{Structure Formation and Properties}

Pulsed laser irradiation has produced a variety of periodic micrometer and sub-micrometer scale surface patterns including laser induced periodic surface structures (LIPSS) when irradiated with nanosecond and femtosecond laser pulses (Dolgaev et al., 2001; Fauchet \& Siegman, 1982; Her et al., 1998; Her et al., 2000; Pedraza et al., 1999; Rothenberg \& Kelly, 1984; Sipe et al., 1983) and with the fluence near the melting threshold. The mechanism responsible for the formation of LIPSS is now well understood. There are reports (Clapham \& Hutley, 1973; Dingley \& Field, 1997; Henry et al., 1998; Her et al., 1998; Her et al., 2000; Pedraza et al., 1999; Rothenberg \& Kelly, 1984; Shen et al., 2008; Vorobyev \& Guo, 2006, 2010, 2011; Vorobyev et al., 2007; Wang \& Guo, 2005, 2006) of surface structures produced on semiconductors, metals, thin films and dielectrics after irradiation with pulsed femtosecond lasers.

\subsection{Silicon}

The formation of nano- and micro-structures on silicon surfaces with femtosecond laser irradiation have been extensively studied (Crouch et al., 2004; Her et al., 1998, 2000; Shen et al., 2003). Femtosecond laser pulses above the ablation threshold in $\mathrm{SF}_{6}$ or $\mathrm{Cl}_{2}$ at a pressure of 500-Torr were used to irradiate and create sharp semi-ordered conical microspikes on the surfaces of silicon (Her et al., 1998, 2000), shown in Figure 1 without the use of masks and with a mask shown in Figure 2 (Shen et al., 2003). The spikes produced are roughly aligned in rows with their direction always coinciding with the direction of the laser light and independent of gravity. They showed that the laser fluence, the pulse duration and the spatial profile had a major effect on the size, height and spatial distribution of the spikes. Spikes of height $40 \mu \mathrm{m}$ and base cross sectional area of about $6 \times 10 \mu \mathrm{m}^{2}$ which tapers down to a diameter of about $0.8 \mu \mathrm{m}$ near the tip were produced as shown in Figure 1. The role of the background gas was also studied and it was found that the spikes are produced in $\mathrm{SF}_{6}, \mathrm{H}_{2} \mathrm{~S}$, or $\mathrm{Cl}_{2}$ but not in $\mathrm{N}_{2}$, vacuum or $\mathrm{He}$ (Carey, 2004) as can be seen in Figure 3. The spikes produced in $\mathrm{SF}_{6}$, or $\mathrm{Cl}_{2}$ had spherical caps at the tips. In $\mathrm{N}_{2}$, vacuum or He blunt and not sharp spikes are formed. This suggests that the background gas is essential to their formation. They explained the spike formation as due to supercooled liquid silicon as the recrystallization front propagates towards the surface at different rates in different locations (Carey, 2004). To better understand the mechanism of microspike formation, Shen and his colleagues (Shen et al., 2003) imposed boundary conditions on the excited silicon surface and the incident laser light by irradiating the silicon surface through a periodic mask in $\mathrm{SF}_{6}$ at a translation speed of $200 \mu \mathrm{m}$ at a fluence of $6 \mathrm{~kJ} / \mathrm{m}^{2}$ well above the ablation threshold. They identified three stages in the spike formation process. LIPSS formed with the same wavelength as the laser, which then coarsen on top of the ripples and then finally fragment into beads which later etch into microspikes.

The formation of high-density regular arrays of nanometer-scale rods on the surface of silicon immersed in water after femtosecond laser irradiation has also been studied (Shen et al., 2008). The sample was translated in a direction perpendicular to the laser beam at a speed of $500 \mu \mathrm{m} / \mathrm{s}$, resulting in an average irradiation of 200 laser pulses at a repetition rate of $1 \mathrm{kHz}$ at a fluence of $2-4 \mathrm{~kJ} / \mathrm{m}^{2}$. To produce the nanorods shown in Figure 4, the sample was irradiated a second time in water at a fluence of $0.5 \mathrm{~kJ} / \mathrm{m}^{2}$ with the laser polarization rotated by $90^{\circ}$ so that it became parallel with the long axis of the ripples. This two-step irradiation results in a surface that is uniformly covered with nanometer-scale rods about $400 \mathrm{~nm}$ high and $50 \mathrm{~nm}$ in diameter. The ejected ablation plume (Tull et al., 2006) from the femtosecond laser ablation of silicon in the presence of $\mathrm{H}_{2}$ or $\mathrm{H}_{2} \mathrm{~S}$ has also been studied.

The presence of the background gas results in the suspension of the plume material for a long period in the chamber, which results in the formation of long straight lines and webs of nanoparticles. The production of superhydrophobic silicon surfaces have also been studied (Baldacchini et al., 2006). They microstructured silicon surfaces with 
femtosecond laser irradiation and which surfaces were later coated with a layer of fluoroalkylsilane molecules to produce superhydrophobic surfaces. In Sheehy et al. (2007) the doping of silicon with selenium and tellurium were studied. Powder of each dopant was spread on a silicon substrate and irradiated with femtosecond laser pulses, examined and compared the resulting morphology, optical properties and chemical composition for each doped substrate before and after thermal annealing. Guo and his colleague (Vorobyev \& Guo, 2010) also used highintensity femtosecond laser pulses to transform regular silicon to superwicking (Vorobyev \& Guo, 2011) and produced black silicon by using femtosecond laser irradiation to structure silicon surface with a grating of equallyspaced parallel nanostructure-textured microgrooves.
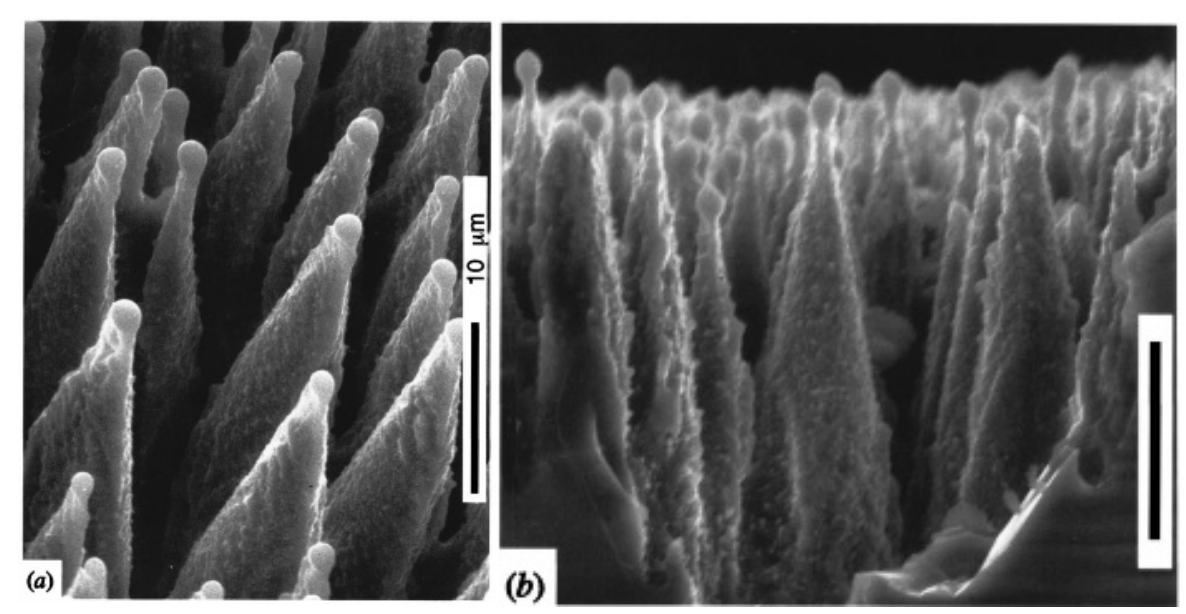

Figure 1. Sharp conical spikes produced on $\mathrm{Si}(100)$ by 500 laser pulses of $100 \mathrm{fs}$ duration, $10 \mathrm{~kJ} / \mathrm{m}^{2}$ fluence in $\mathrm{SF}_{6}$ at a pressure of 500 Torr viewed (a) $45^{\circ}$ from the surface normal, and (b) parallel to the surface (From (Her et al., 1998)).

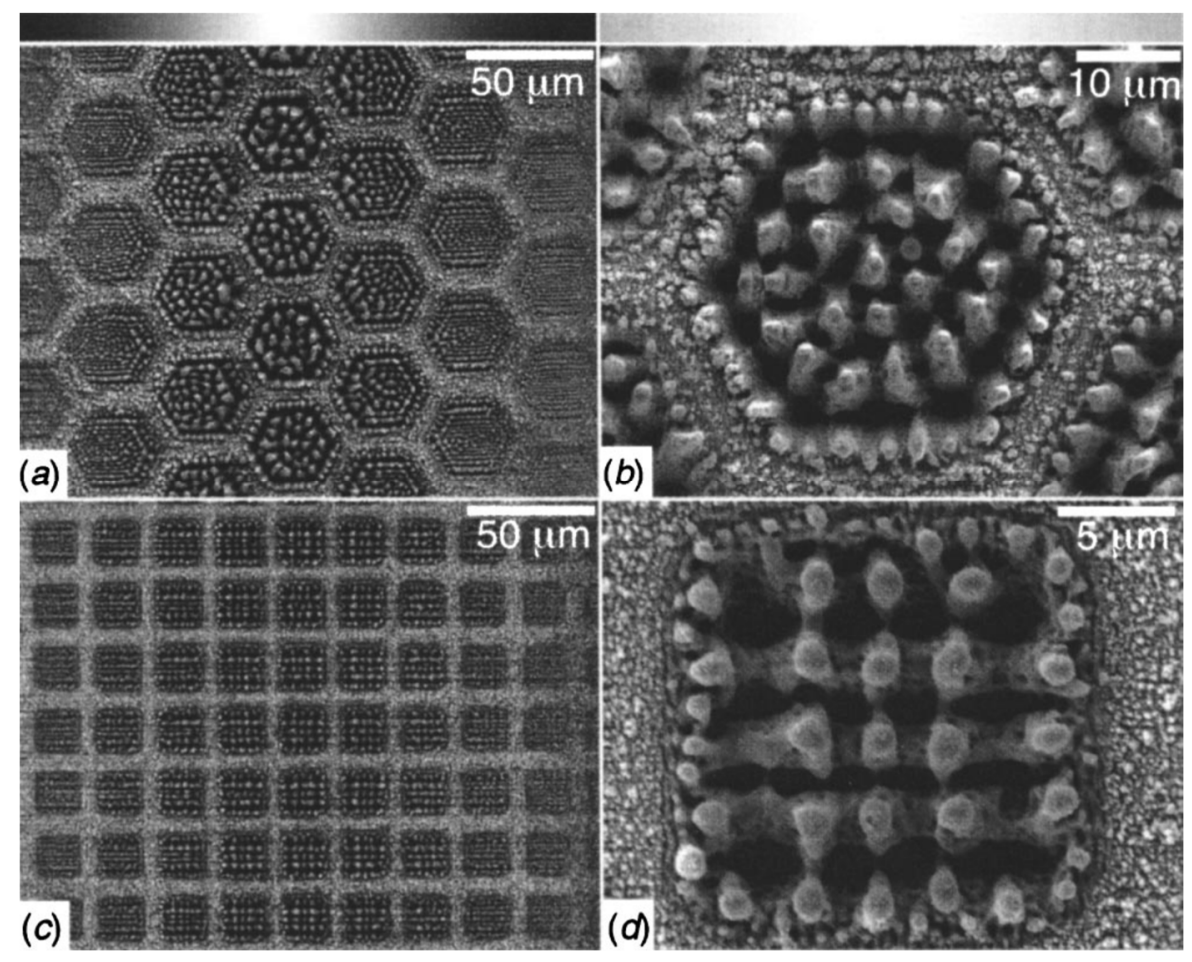

Figure 2. Scanning electron micrographs of ordered silicon spikes formed by masking the irradiated sample with (a), (b) a $30 \mathrm{~mm}$ hexagonal grid and (c), (d) a $20 \mathrm{~mm}$ square grid. The nearly Gaussian spatial intensity profile of the laser pulse is shown at the top in grayscale (white corresponds to maximum intensity) (From (Shen et al., 2003)). 

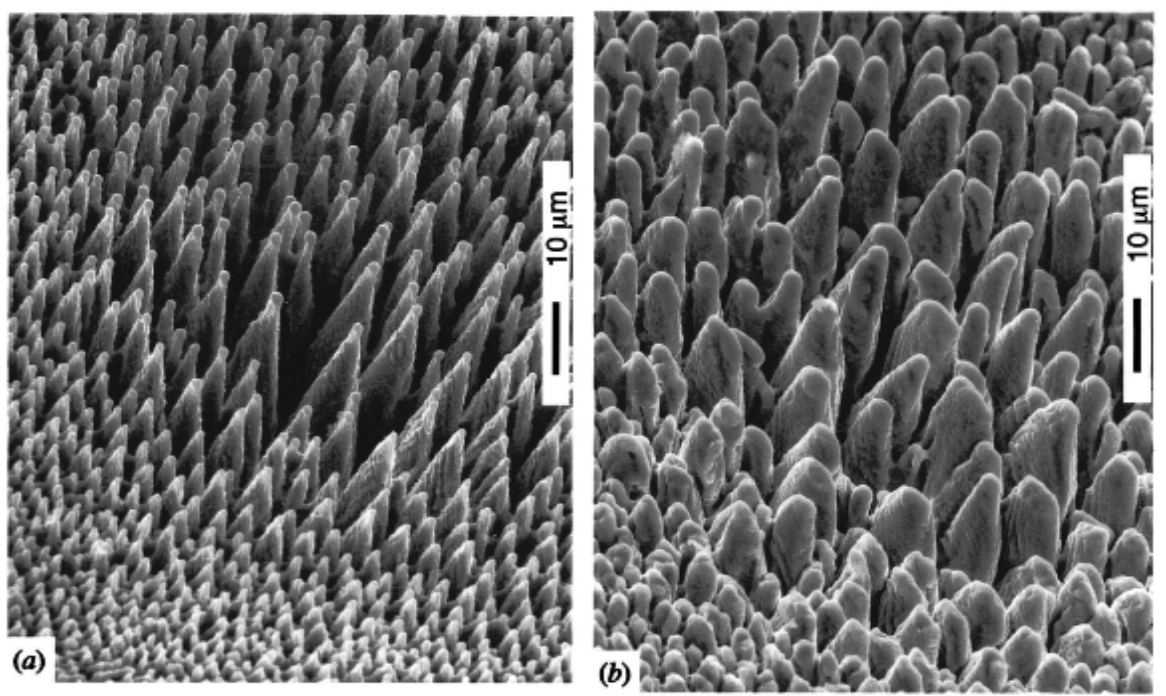

Figure 3. Comparison of $\mathrm{Si}(100)$ surfaces irradiated in the presence of (a) $\mathrm{SF}_{6}$ and (b) vacuum viewed at an angle of $45^{\circ}$ from the surface normal. The laser spot size is $200 \mu \mathrm{m}$ in diameter (From (Her et al., 1998)).

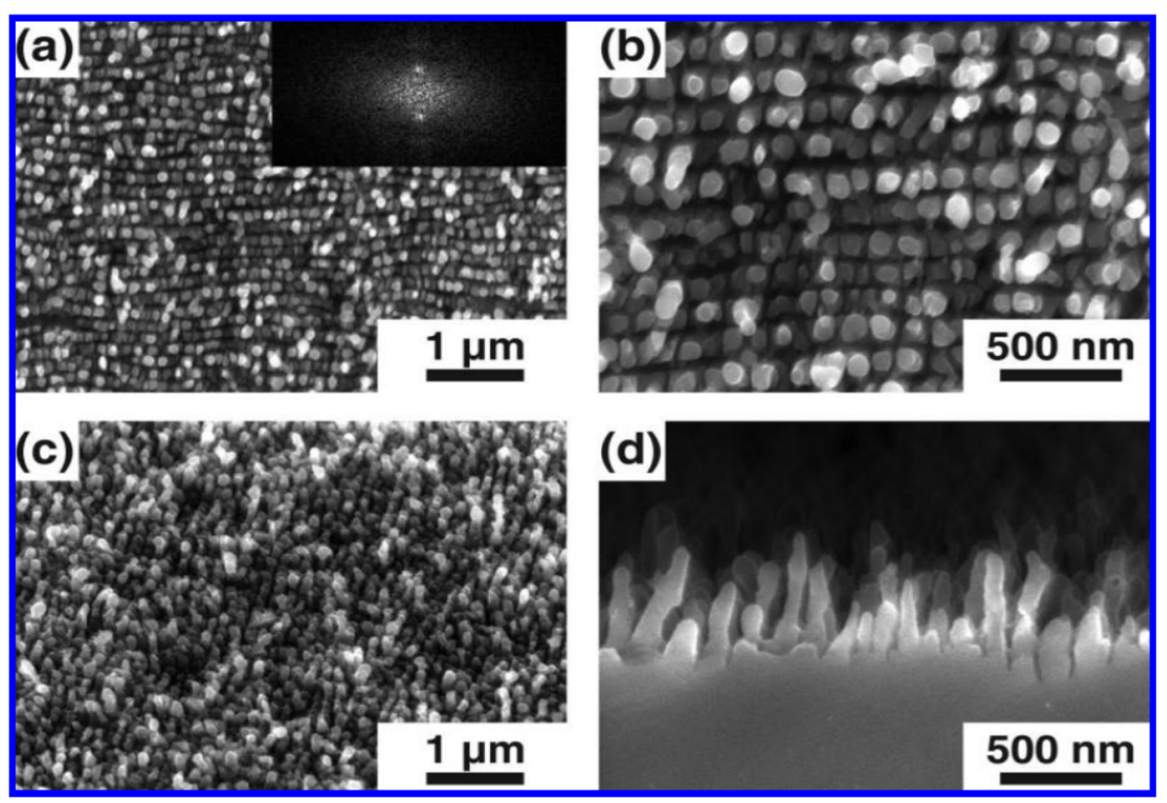

Figure 4. SEM pictures of nanometer-scale rods formed using a two-step irradiation process of a silicon surface: the nanoripples are irradiated a second time in water at a fluence of $0.5 \mathrm{~kJ} / \mathrm{m}^{2}$ by an average of $200800 \mathrm{~nm}$ laser pulses, with the polarization rotated by $90^{\circ}$. (a, b) Top view, (c) $45^{\circ}$ view, and (d) cross-sectional side view of the nanometer-scale rods. The inset of (a) shows a Fourier transform of the image in (a) (From Shen et al., 2008)

\subsubsection{Spike Formation}

The influence of the ambient gas on the shape of the spikes indicates that chemical reaction between $\mathrm{SF}_{6}$ or $\mathrm{Cl}_{2}$ and silicon contributes to the formation of the sharp spikes (Raksi et al., 1996). This is because sharp spikes are not formed in $\mathrm{He}$ or $\mathrm{N}_{2}$ and also not in vacuum suggesting their role is not purely physical. Femtosecond laser pulses can dissociate $\mathrm{SF}_{6}$, and silicon may be etched by the resulting radicals to form volatile $\mathrm{SiF}_{4}$. The laser pulses could also produce vibrationally excited $\mathrm{SF}_{6}$, which has an enhanced reactivity with the silicon surface. The suggested alternative route is the melting of silicon, followed by $\mathrm{Cl}_{2}$ etching (Chuang, 1981; Pronko et al., 1995; Raksi et al., 1996; Sokolowski-Tinten et al., 1995). They performed backscattering Kikuchi diffraction on the spikes which show they are crystalline with the same structure as bulk silicon (Dingley \& Field, 1997; Her et al., 
2000). They also found a correlation between both the microstructure height and distance between microstructures in equation (1) as

$$
h \propto d^{2.4 \pm 0.1}
$$

where $h$ and $d$ are microstructure height and separation distance respectively (Carey, 2004; Her et al., 2000).

The ripples formed by femtosecond lasers when masks were used can be likened to those formed in the nanosecond case where ripple formation is due to capillary waves excited in the molten silicon by interference between the incident and the scattered laser light (Sheehy et al., 2005; Shen et al., 2003). Fresnel diffraction of the incident light from the edges of the mask openings should also produce a modulated temperature profile in the plasma or the liquid. But since increasing the fluence increases both the typical separation of the microspikes and the wavelength of the coarsened layer, plasma oscillations cannot be the mechanism responsible for the arrangement of the microspikes (Shen et al., 2003). For capillary waves to be responsible for the formation mechanism, they saw that diffraction of the laser light from the mask apertures causes inhomogeneous energy deposition into the substrate resulting in the temperature in the molten surface layer being periodically modulated (Shen et al., 2003).

\subsubsection{Light Absorption}

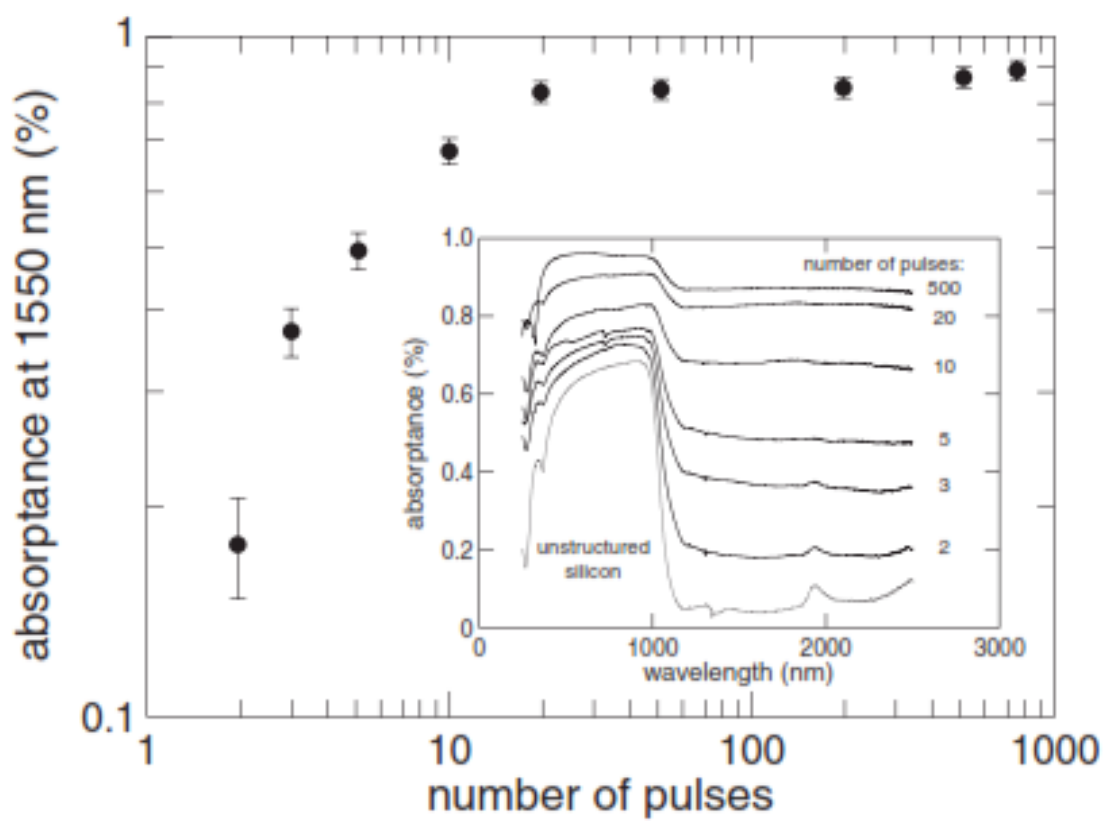

Figure 5. Dependence of the absorance at $1550 \mathrm{~nm}$ of microstructured silicon surfaces on the number of laser pulses used in microstructuring. All samples were made with $8 \mathrm{~kJ} / \mathrm{m}^{2}$ laser fluence and $0.67 \mathrm{bar} \mathrm{SF} 6$ pressure.

Inset: Wavelength dependence of absorance for a subset of the samples shown in Figure 3 and for the unstructured silicon substrate (From (Crouch et al., 2004)).

Infrared radiation absorption by microstructured silicon surfaces has been extensively studied based on the effect of processing conditions and anneal temperature (Carey, 2004; Crouch et al., 2004; Her et al., 1998; Sheehy et al., 2005) and metal film on the microstructured silicon surface (Wang et al., 2009). Crouch and colleagues (Crouch et al., 2004) microstructured silicon in the presence of $\mathrm{SF}_{6}$ and found that the microstructured sample exhibited near unity below band gap absorption. The absorance depended on the experimental parameters: number of pulses, laser fluence, background gas pressure, background gas and anneal temperature.

They studied the influence of each of these parameters with the others kept constant at a wavelength of $1550 \mathrm{~nm}$. The absorptance at $1550 \mathrm{~nm}$ increased with increasing number of pulses and peaked at 500 pulses as shown in Figure 5. The absorption also increased with laser fluence and peaked at $12 \mathrm{~kJ} / \mathrm{m}^{2}$ as shown in Figure 6 , and also with $\mathrm{SF}_{6}$ pressure where it peaked at a pressure of 0.67 bar (Crouch et al., 2004). To make it clear that it was the presence of sulfur in the background gas that was responsible for the increase of absorptance in the below band gap region, Carey (2004) used $\mathrm{H}_{2} \mathrm{~S}$ as the ambient gas, which also increased the absorptance. The absorptance at 
$1550 \mathrm{~nm}$ however decreased with the anneal temperature from room temperature to $875 \mathrm{~K}$. Sheehy and his colleagues (Sheehy et al., 2005) also found that silicon samples microstructured in $\mathrm{N}_{2}$, air and $\mathrm{Cl}_{2}$ showed enhanced optical absorptance for above-band gap radiation, but had an absorptance that decreased monotonically from the band edge of $1.1 \mu \mathrm{m}$ to $2.5 \mu \mathrm{m}$. The surfaces they microstructured in $\mathrm{SF}_{6}$ or $\mathrm{H}_{2} \mathrm{~S}$ under the wide range of processing conditions above exhibited strong, featureless below band gap absorptance from 1.2 to $2.5 \mu \mathrm{m}$ but the absorptance was affected by annealing. Under these processing conditions and annealing, the visible absorptance of the samples was not affected (Carey, 2004; Janzen et al., 1984). These optical properties suggest that the band structure is altered with a reduced band gap and that the microstructured material has a diodic $I-V$ characteristic and high photoresponse to wavelength longer than $1.1 \mu \mathrm{m}$. This indicates that the band gap of the microstructured surface layer is different from that of the bulk (Carey, 2004). These authors have attributed the altered band structure to the formation of an impurity band due to the presence of high concentrations of sulfur in the nanocrystalline layer at the surface of the sample (Crouch et al., 2004; Sheehy et al., 2005). Silicon has such a small absorption coefficient in the near infrared that multiple reflections on microstrucutred sidewalls alone cannot account for this below band gap absorptance. Damage, disorder and incorporation of sulfur creates a tail of states below the band gap by changing the bond lengths, bond angles, and/or coordination of the silicon. These changes to the silicon lattice explains the below band gap absorptance in $\mathrm{SF}_{6}$ or $\mathrm{H}_{2} \mathrm{~S}$ (Liu et al., 2008; Sheehy et al., 2005).

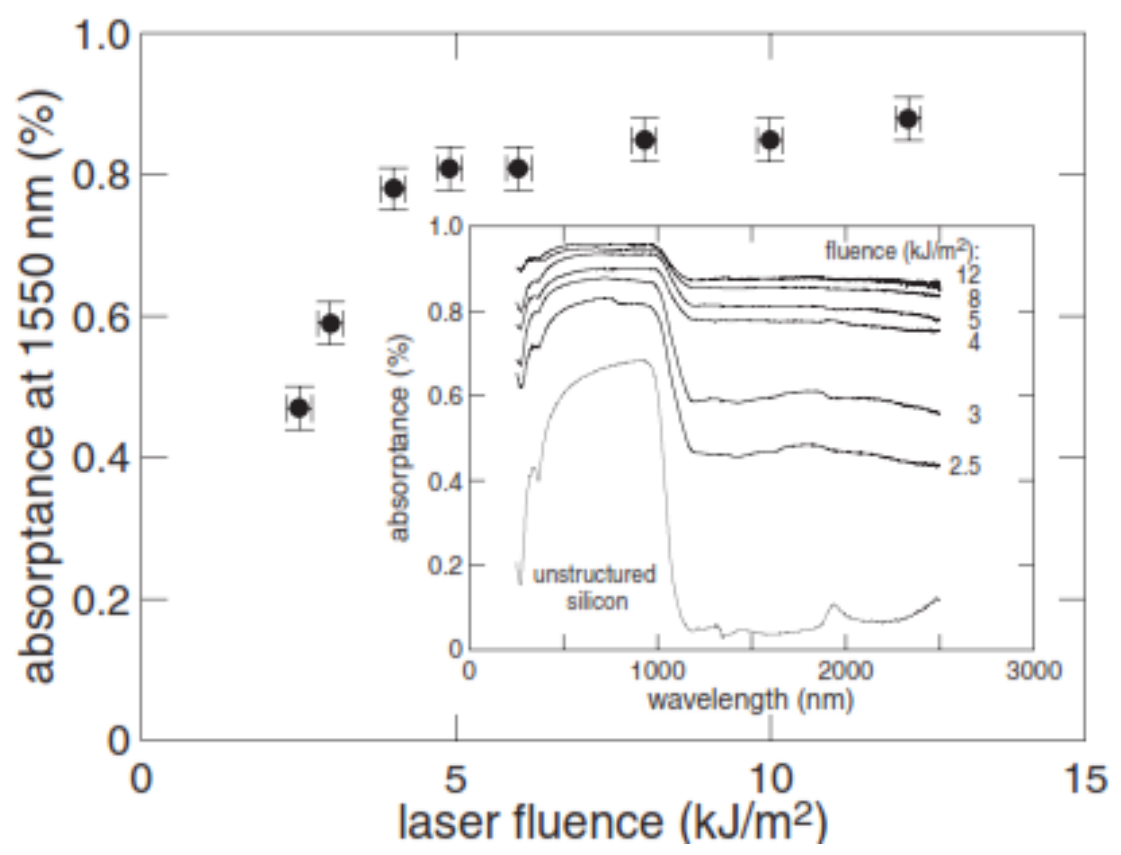

Figure 6. Dependence of the absorptance at $1550 \mathrm{~nm}$ of microstructured silicon surfaces on the laser fluence used in microstructuring. All samples were made with 500 laser pulses and 0.67 bar $\mathrm{SF}_{6}$ pressure. Inset: Wavelength dependence of the absorptance for a subset of the samples shown in Figure 4 and for the unstructured silicon substrate (From Crouch et al., 2004).

\subsection{Metal}

The experiments (Vorobyev \& Guo, 2005) were performed in air and mechanically polished gold was used. The authors studied the optical properties of the surface modifications following multipulse ablation at single-pulse laser fluences of $F=1.1,0.35,0.17$, and $0.078 \mathrm{~J} / \mathrm{cm}^{2}$ in air. This is shown in Figure 7. They found that the ablation thresholds $F_{a b l}$ for a pristine surface were $F_{a b l}=0.067$ and $0.048 \mathrm{~J} / \mathrm{cm}^{2}$ for single- and 500-pulse train irradiation, respectively (Vorobyev \& Guo, 2005). To study the absorptance of an ablated spot on the sample after a chosen number of pulses, the authors reduced the laser fluence used initially to a level much below the ablation threshold. The ablated spot is subsequently irradiated again with a train of low fluence laser pulses that will not induce any further surface modification. Through a series of measurements, a study was conducted (Vorobyev \& Guo, 2005) which determined the absorptance $\mathrm{A}$ of the ablated spot as expressed in equation (2) as: 


$$
A=E_{A} / E_{l}
$$

where $E_{A}=C \Delta T$ is the amount of energy from the low fluence pulse and causes a temperature rise of $\Delta \mathrm{T}$ in the skin layer of the sample; $\mathrm{C}$ is the heat capacity of the sample. $\mathrm{E}_{1}$ is the estimated incident laser energy upon the sample (Vorobyev \& Guo, 2005).

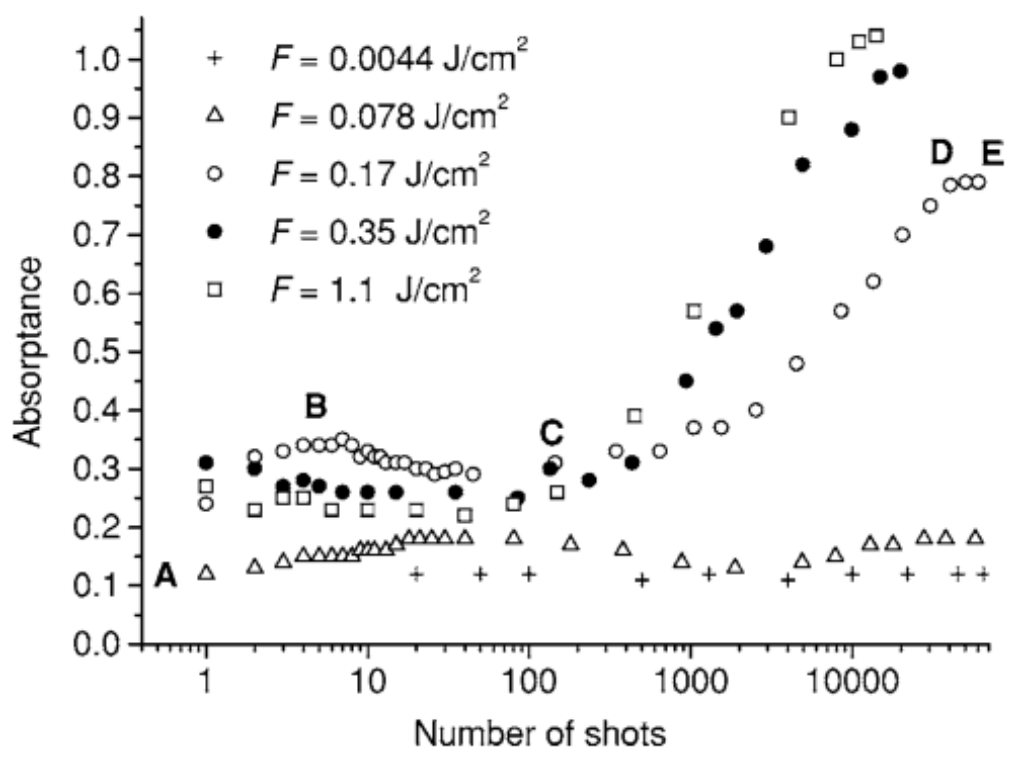

Figure 7. Absorptance of gold surface following femtosecond laser ablation as a function of the number of pulses at various fluencies $F$. The measured absorptance is slightly higher than 1 at $F=1.1 \mathrm{~J} / \mathrm{cm}$ (From (Vorobyev \&

Guo, 2005)).

The study concluded that the absorptance of gold increased significantly at high fluence and large number of applied pulses (Vorobyev \& Guo, 2005). Wang and his colleague (Wang \& Guo, 2006) studied type- $c$ structures which are a variation of LIPSS on metals with a $p$-polarized femtosecond laser irradiation. At a range of incident angles larger than $50^{\circ}$, uniform type- $c$ structures are formed on $\mathrm{Cu}$ with fluencies just above the damage threshold. But for gold and silver, type- $c$ structures only occured at fluencies far above the damage threshold and not close to it. The clearness level of periodic surface patterns of $\mathrm{Cu}, \mathrm{Au}$, and $\mathrm{Ag}$ have been determined (Wang \& Guo, 2005) using $p$-polarized femtosecond laser irradiation. A detailed study of surface structural modifications on femtosecond laser ablated gold and platinum at near-damage-threshold fluence was conducted (Vorobyev et al., 2007) and was found that the period of the nanostructure that covered LIPSS was appreciably less than that of the regular LIPSS. A horizontally polarized femtosecond laser beam has been used to create surface patterns on metals and their wetting properties studied (Vorobyev \& Guo, 2009). They found that liquid runs vertically uphill against gravity on the structured surfaces.

\subsubsection{Structure Formation}

In order to determine the mechanism of nanostructuring, Vorobyev \& Guo (2006) made a careful SEM study of nascent nanostructures following ablation with fluence of $35 \mathrm{~J} / \mathrm{m}^{2}$ and 1 pulse and compared it with an undamaged area of the sample using the same scale. They found from the comparison that surface structuring was initiated on random highly localized nano-scale sites which typically included nanopores with diameter in the range of 40-100 $\mathrm{nm}$, randomly oriented nanoprotrusions with a diameter in the range of $20-70 \mathrm{~nm}$ and a length of $20-80 \mathrm{~nm}$, nanocavities of arbitrary form, and nanorims around nanocavities. When the fluence was close to the ablation threshold, the spatial variations in deposited laser energy produced a melt at localized nanoscale sites within the irradiated spot. A high radial temperature gradient in a nanomelt could induce a radial surface tension gradient that expeled the liquid to the periphery of the nanomelt (Bass \& Liou, 1984; Vorobyev \& Guo, 2006), leading to the formation of nanocavities, nanoprotrusions, and nanorims. These initially induced surface random nanostructures could enhance the absorption of laser light (Vorobyev \& Guo, 2005). SEM morphology studies at high fluence showed that melt occured over a large area of the ablated spot and the flow dynamics in this large pool of melt predominantly resulted in microstructuring (Vorobyev \& Guo, 2006). 


\subsubsection{Light Absorption}

The optical properties of surface modifications have been studied by Vorobyev \& Guo (2005) following multipulse ablation at single-pulse laser fluencies of $F=1.1,0.35,0.17$, and $0.078 \mathrm{~J} / \mathrm{cm}^{2}$ in air. From their measurements it was concluded that the absorptance remained constant when measured at a fluence below the ablation threshold, but the absorptance of a structurally modified surface was significantly greater than that of the undamaged surface and strongly depended on the applied ablation pulses N (Figure 7) (Vorobyev \& Guo, 2005). The findings were that the physical mechanism of the enhanced absorption was due to a combined effect of nanostructural, microstructural, and macrostructural surface modifications induced by femtosecond laser ablation. Another study (Ang et al., 1997; Kreibig \& Vollmer, 1995; Vorobyev \& Guo, 2005) concluded that surface roughness could enhance the absorption of light both by multiple reflections in microcavities and by variation in the angle of incidence (Granqvist \& Hunderi, 1977; Prokhorov et al., 1990). Laser-induced periodic surface structures (LIPSS) may enhance absorption of laser energy via generation of surface electromagnetic waves (Ursu et al., 1985). Since redeposition of ablated material occured both outside and within the ablated spot, the redeposition of the nanoparticles produced by ablation could also enhance the absorption of light in the ablated area (Vorobyev \& Guo, 2005).

\section{Summary}

In this review representative results of material ablation and surface structuring by femtosecond laser pulsed irradiations have been appraised. The emphasis was on infrared light absorption of these materials after surface structuring with the materials considered being silicon and metals $(\mathrm{Au}, \mathrm{Al})$. The experimental technique was femtosecond laser irradiation and with varied experimental conditions including structuring in air, $\mathrm{SF}_{6}, \mathrm{CL}_{2}, \mathrm{~N}_{2}$ or other gases, or in water. Depending on the experimental conditions microspikes, blunt microstrucutres, nanostructures could be formed on the samples. The structures on the metals also depend on the polarization of the laser while the absorption of infrared radiation is enhanced on the structured surfaces depending on the experimental conditions. This opens up a new pathway towards infrared light detection.

Considering the enhancement of light absorption in transparent materials it will be interesting after this study on femtosecond laser irradiation to study light absorption in thin films of these materials after laser structuring. Even though some femtosecond laser structuring experiments with metal films have been reported, these studies did not focus on light absorption. Specifically with the study involving infrared light absorption (Wang et al., 2009) the metal films were deposited on the structured silicon. It will be informative to study the absorption properties of very thin films of metals deposited on transparent materials (on either the surface or back surface) and then microstructured with femtosecond laser irradiation. Plasmonic studies can also be undertaken on a transparent material when one surface is microstructured and metal thin films deposited on the other surface since structuring the surface increases the light absorption and localized surface plasmons also increases absorption.

\section{Conflict of interests}

The authors declare that there is no conflict of interests regarding the publication of this paper.

\section{References}

Allmen, M. F. V., \& Lau, S. S. (1982). In Laser Annealing of Semiconductors (Eds.). New York: Academic.

Ang, L. K., Lau, Y. Y., Gilgenbach, R. M., \& Spindler, H. L. (1997). Analysis of laser absorption on a rough metal surface. Applied Physics Letters, 70, 696-698.

Baldacchini, T., Carey, J. E., Zhou, M., \& Mazur, E. (2006). Superhydrophobic Surfaces prepared by microstructuring of silicon using a femtosecond laser. Langmuir, 22, 4917-4919.

Bass, M., \& Liou, L. (1984). Calorimetric studies of light absorption by diamond turned $\mathrm{Ag}$ and $\mathrm{Cu}$ surfaces and analyses including surface roughness contributions. Journal of Applied Physics, 56, 184-184.

Brorson, S. D., Kazeroonian, A., Moodera, J. S., Face, D. W., ..., \& Dresselhaus, G. (1990). Femtosecond roomtemperature measurement of the electron-phonon coupling constant $\gamma$ in metallic superconductors. Physical Review Letters, 64, 2172-2175.

Carey, J. E. (2004). Femtosecond Microstructuring of Silicon for Novel Optoelectronic Devices Harvard University, Cambridge.

Chuang, T. J. (1981). Multiple photon excited $\mathrm{SF}_{6}$ interaction with silicon surfaces. Journal Chemical Physics, 74, $1453-1460$. 
Clapham, P. B., \& Hutley, M. C. (1973). Reduction of Lens Reflexion by the "Moth Eye" Principle. Nature, 244, 281-282.

Crouch, C. H., Carey, J. E., Shen, M., Mazur, E., \& Genin, F. Y. (2004). Infrared absorption by sulfur-doped silicon formed by femtosecond laser irradiation. Applied Physics A, 79, 1635-1641.

Crouch, C. H., Carey, J. E., Warrender, J. M., Aziz, M. J., \& Mazur, E. (2004). Comparison of structure and properties of femtosecond and nanosecond laser-structured silicon. Applied Physics Letters, 84, 1850-1852.

Dam, B., Rector, J. H., Johansson, J., Kars, S., \& Griessen, R. (1996). Stoichiometric transfer of complex oxides by pulsed laser deposition. Applied Surface Science, 96/98, 679-684.

Dingley, D. J., \& Field, D. P. (1997). Electron Backscatter Diffraction and Orientation Imaging Microscopy. Material Science Technology, 13, 69-78.

Dolgaev, S. I., Lavrishev, S. V., Lyalin, A. A., Simakin, A. V., Voronov, V. V., \& Shafeev, G. A. (2001). Formation of conical microstructures upon laser evaporation of solids. Applied Physics A: Material Science Processing, 73, 177-181.

Eesley, G. L. (1986). Generation of nonequilibrium electron and lattice temperatures in copper by picosecond laser pulses. Physical Review, 33, 2144-2151

Fauchet, P. M., \& Siegman, A. E. (1982). Surface ripples on silicon and gallium arsenide under picosecond laser illumination. Applied Physics Letters, 40, 824-826.

Granqvist, C. G., \& Hunderi, O. (1977). Optical properties of ultrafine gold particles. Physics Review B, 16, 35133534.

Henry, S., McAllister, D. V., Allen, M. G., \& Prausnitz, M. R. (1998). Microfabricatedmicroneedles: A novel approach to transdermal drug delivery. Journal of Pharmaceutical Sciences, 87, 922-925.

Her, T. H., Finlay, R. J., Wu, C., \& Mazur, E. (2000). Femtosecond laser-induced formation of spikes on silicon. Applied Physics A, 70, 383-385.

Her, T. H., Finlay, R. J., Wu, C., Deliwala, S., \& Mazur, E. (1998). Microstructure of silicon with femtosecond laser pulses. Applied Physics Letters, 73, 1673-1675.

Hirayama, Y., \& Obara, M. (2002). Heat effects of metals ablated with femtosecond laser pulses. Applied Surface Science, 197/198, 741-745.

Janzen, E., Stedman, R., Grossmann, G., \& Grimmeiss, H. G. (1984). High-resolution studies of sulfur- and selenium-related donor centers in silicon. Physics Review B, 29, 1907-1918.

Jhee, Y. K., Becker, M. F., \& Walser, R. M. (1985). Charge emission and precursor accumulation in the multiplepulse damage regime of silicon. Journal of Optical Society America B, 2, 1626-1633.

Kanemitsu, Y., Nakada, I., \& Kuroda, H. (1985). Picosecond laser induced anomalous crystallization in amorphous silicon. Applied Physics Letters, 47, 939-941.

Kautek, W., \& Krueger, J. (Eds.) (1995). Femtosecond Pulse Laser Microstructuring of Semiconducting Materials. Switzerland: Trans Tech Publications.

Ko, S. H., Choi, Y., Hwang, D. J., \& Grigoropoulos, C. P. (2006). Nanosecond laser ablation of gold nanoparticle films. Applied Physics Letters, 89, 1411261-1411263.

Kreibig, U., \& Vollmer, M. (1995). Optical Properties of Metal Clusters. Berlin: Springer-Verlag.

Le Drogoff, B., Margot, J., Chaker, M., Sabsabi, M., ..., \& von Kaenel, Y. (2001). Temporal characterization of femtosecond laser pulses induced plasma for spectrochemical analysis of aluminum alloys. Spectrochim. Acta Part B, 56, 987-1002.

Liu, Y., Liu, S. Y., Wang, Y., Feng, G. J., Zhu, J. T., \& Zhao, L. (2008). Broad band enhanced infrared light absorption of a femtosecond laser microstructured silicon. Laser Physics, 18, 1148-1152.

Momma, C., Nolte, S., Chichkov, B. N., Alvensleben, F. V., \& Tuennermann, A. (1997). Precise laser ablation with ultrashort pulses. Applied Surface Science, 109/110, 15-19.

Nedialkov, N. N., Atanasov, P. A., Amoruso, S., Bruzzese, R., \& Wang, X. (2007). Laser ablation of metals by femtosecond pulses: Theoretical and experimental study. Applied Surface Science, 253, 7761-7766.

Nissim, Y. I., Sapriel, J., \& Oudar, J. L. (1983). Microprobe Raman analysis of picosecond laser annealed implanted silicon. Applied Physics Letters, 42, 504-506. 
Pedraza, A. J., Fowlkes, J. D., \& Lowndes, D. H. (1999). Silicon microcolumn arrays grown by nanosecond pulsedexcimer laser irradiation. Applied Physics Letters, 74, 2322-2324.

Prokhorov, A. M., Konov, V. I., Ursu, I., \& Mihailescu, I. N. (1990). Laser Heating of Metals. Bristol: Adam Hilger.

Pronko, P. P., Vanrompay, P. A., Singh, R. K., Qian, F., Du, D., \& Liu, X. (1995). Laser induced avalanche ionization and electron-lattice heating of silicon with intense near IR femtosecond pulses. Materials Research Society Symposium Proceedings, 397, 45.

Raksi, F., Wilson, K., Jiang, Z., Ikhlef, A., Cote, C. Y., \& Kieffer, J. (1996). Ultrafast x-ray absorption probing of a chemical reaction. Journal Chemical Physics, 104, 6066-6069.

Rothenberg, J. E., \& Kelly, R. (1984). Laser sputtering. Part II. The mechanism of the sputtering of Al2O3. Nuclear Instruments and Methods in Physics Research Section B, 1, 291.

Schaffer, C. B., Garcia, J. F., \& Mazur, E. (2003). Bulk heating of transparent materials using a high-repetitionrate femtosecond laser. Applied Physics A, 76, 351-354.

Scott, S. L., Crudden, C. M., \& Jones, C. W. (2003). Nanostructured catalysts. New York: Kluwer Academic.

Sheehy, M. A., Tull, B. R., Friend, C. M., \& Mazur, E. (2007). Chalcogen doping of silicon via intense femtosecond-laser irradiation. Material Science and Engineering B, 137, 289-294.

Sheehy, M. A., Winston, L., Carey, J. E., Friend, C. M., \& Mazur, E. (2005). Role of the background gas in the morphology and optical properties of laser-microstructured silicon. Chemistry of Materials, 17, 3582.

Shen, M. Y., Crouch, C. H., Carey, J. E., Younkin, R., \& Mazur, E. (2003). Formation of regular arrays of silicon microspikes by femtosecond laser irradiation through a mask. Applied Physics Letters, 82, 1715-1717.

Shen, M., Carey, J. E., Crouch, C. H., Kandyla, M., Stone, H. A., \& Mazur, E. (2008). High density regular arrays of nanometer-scale rods formed on silicon surfaces via femtosecond laser irradiation in water. Nano Letters, $8,2087-2091$.

Sipe, J. E., Young, J. F., Preston, J. S., \& van Driel, H. M. (1983). Laser-induced periodic surface structure. I. Theory Physics Review B, 27, 1141-1154.

Sokolowski-Tinten, K., Bialkowski, J., \& von der Linde, D. (1995). Ultrafast Laser-Induced Order-Disorder Transitions in Semiconductors. Physics Review B, 51, 14186-14198.

Tull, B. L., Carey, J. E., Sheehy, M. A., Friend, C., \& Mazur, E. (2006). Formation of silicon nanoparticles and web-like aggregates by femtosecond laser ablation in a background gas. Applied Physics A, 83, 341-346.

Ursu, I., Mihailescu, I. N., Prokhorov, A. M., Konov, V. I., \& Tokarev, V. N. (1985). On the role of the periodical structures induced by powerful laser irradiation of metallic surfaces in the energy coupling process. Physica $B \& C, 132 C, 395-402$.

Vorobyev, A. Y., \& Guo, C. (2005). Enhanced absorptance of gold following multipulse femtosecond laser ablation. Physics Review B, 72, 195422.

Vorobyev, A. Y., \& Guo, C. (2006). Femtosecond laser nanostructuring of metals. Optics Express, 14, 2164-2169.

Vorobyev, A. Y., \& Guo, C. (2008). Colorizing metals with femtosecond laser pulses. Applied Physics Letters, 92, 0419141-0419143.

Vorobyev, A. Y., \& Guo, C. (2009). Metal pumps liquid uphill. Applied Physics Letters, 94, 2241021-2241023.

Vorobyev, A. Y., \& Guo, C. (2010). Laser turns silicon superwicking. Optics Express, 18, 6455.

Vorobyev, A. Y., \& Guo, C. (2011). Direct creation of black silicon using femtosecond laser pulses. Applied Surface Science, 257, 7291-7294.

Vorobyev, A. Y., Makin, V. S., \& Guo, C. (2007). Periodic ordering of random surface nanostructures induced by femtosecond laser pulses on metals. Journal of Applied Physics, 101, 0349031-0349034.

Vorobyev, A. Y., Makin, V. S., \& Guo, C. (2009). Bright light sources from black metals: Significant increase in emission efficiency of incandescent light sources. Physical Review Letters, 102, 2343011-2343014.

Wang, J., \& Guo, C. (2005). Ultrafast dynamics of femtosecond laser-induced periodic surface pattern formation on metals. Applied Physics Letters, 87, 2519141-2519143. 
Wang, J., \& Guo, C. (2006). Formation of extraordinarily uniform periodic structures on metals induced by femtosecond laser pulses. Journal of Applied Physics, 100, 0235111-0235114.

Wang, Y., Liu, S., Wang, Y., Feng, G., Zhu, J., \& Zhao, L. (2009). Infrared light absorption of silver film coated on the surface of femtosecond laser microstructured silicon in SF6. Material Letters, 63, 2718-2720.

Wellershoff, S. S., Hohlfeld, J., Guedde, J., \& Matthias, E. (1999). The role of electron-phonon coupling in femtosecond laser damage of metals. Applied Physics A, 69(Supl.), S99-S107.

Young, J. F., Preston, J. S., van Driel, H. M., \& Sipe, J. E. (1983). Laser-induced periodic surface structure. II. Experiments on Ge, Si, Al, and brass. Physics Review B, 27, 1155-1172.

\section{Copyrights}

Copyright for this article is retained by the author(s), with first publication rights granted to the journal.

This is an open-access article distributed under the terms and conditions of the Creative Commons Attribution license (http://creativecommons.org/licenses/by/4.0/). 